Case Report,

\title{
Tuberculous Spondylitis in Siti Rahmah Hospital during Covid-19 Pandemic
}

\author{
Yuri Haiga ${ }^{1}$ \\ ${ }^{1}$ Department of Neurology, Baiturrahmah University/ Siti Rahmah Hospital, Padang \\ Email Address: yurihaiga@fk.unbrah.ac.id
}

\begin{abstract}
:
Background:

Spondylitis TB is an infection of Mycobacterium TB involving the spine. The course of spondylitis TB is relatively indolent. This study was conducted to evaluate the clinical presentation and a goal of surgery in two patients who had been operated for spondylitis tuberculosis (TB) in Siti Rahmah Hospital during Covid19 pandemic.
\end{abstract}

\section{Case Report:}

Case 1, a 24-year-old woman, presented to the emergency department with a complaint of nausea, vomiting, weight loss, and severe back pain. On admission she was febrile and had leukocytosis. Initial spinal x-ray was performed and revealed osteolytic changes in the vertebral body of T10 and T11. Magnetic resonance imaging (MRI) of the spine illustrated spondylitis of T8 until T12, with paravertebral abscesses, which was suggestive spondylitis. Case 2, A 19-year-old woman, presented to policlinic with complain of paraplegi inferior and hipoestesi since 7 month ago. Magnetic resonance imaging (MRI) of the spine illustrated spondylitis of C7 until T4, with paravertebral abscesses, which was suggestive spondylitis. Both patients were operated on by an orthopedic doctor. Anatomical Pathology examination showed tuberculosis spodylitis. The patient treat surgical intervention and anti-tuberculosis drug. After surgical intervention, patient had improvement of sensoric and motoric.

\section{Conclusions:}

In a covid-19 pandemic situation, diagnosis and management of spondylitis TB must be carried out because it is related to the prognosis of the disease.

Keywords: Mycobacterium tuberculosis; Spondylitis, Covid-19 Pandemic

\section{Background/objectives:}

Tuberculous spondylitis is an infection of Mycobacterium TB involving the spine. The higher incidence and prevalence of tuberculosis is a common health problem particularly in developing countries. Spinal tuberculosis usually represents at advanced levels and diagnosis of this disease is not easy. Patients with spinal tuberculosis usually represent with gibbus formation, back ache, low grade fever, neurological symptoms and decificts. Although, commonly seen in dorsal spine lesions, cervical and cervico-thoracic lesions with spine tuberculosis rarely seen in literature. The course of tuberculous spondylitis is relatively indolent. This study was conducted to evaluate the clinical presentation and a goal of surgery in two patients who had been operated for tuberculous spondylitis in Siti Rahmah Hospital during Covid-19 pandemic.

\section{Case report:}

Two cases reports of Tuberculous Spondylitis.

Case 1, A 24-year-old woman, presented to the emergency department with a complaint of nausea, vomiting, weight loss, and severe back pain. On admission she was febrile and had leukocytosis. Negative rapid test results. Initial spinal $x$-ray was performed and revealed osteolytic changes in the vertebral body of $\mathrm{T} 10$ and T11. Magnetic 
resonance imaging (MRI) of the spine illustrated spondylitis of T8 until T12, with paravertebral abscesses, which was suggestive spondylitis.

Case 2, A 19-year-old woman, presented to polyclinic with complain of paraplegi inferior and hipoestesi since 7 month ago. Negative rapid test results. MRI of the spine illustrated spondylitis of C7 until T4, with paravertebral abscesses, which

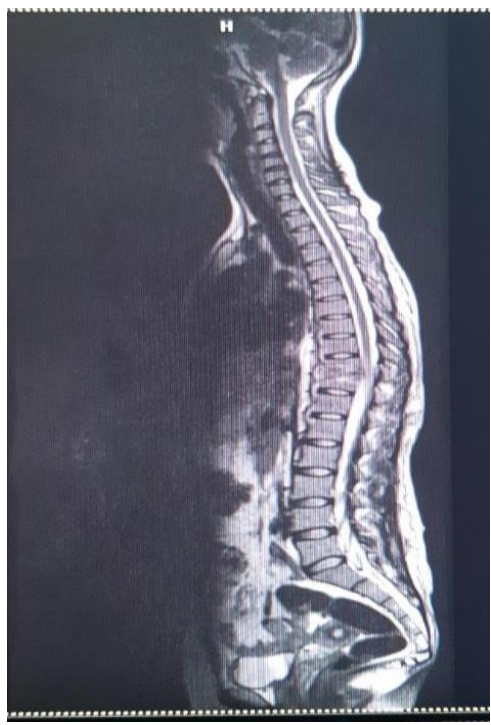

Figure (A). MRI case I,

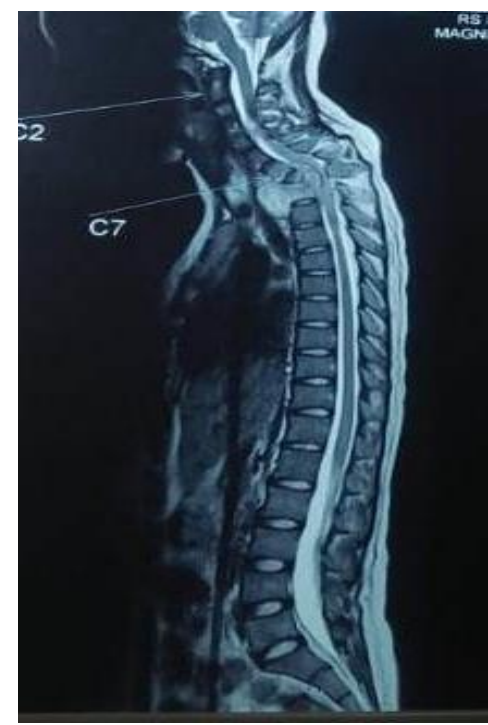

Figure (B) MRI Case II

\section{Results:}

The two cases of tuberculous spondylitis described had different clinical characteristics, and outcomes. Commonly, in tuberculous spondylitis, the symptoms develop insidiously due to the slow progression of the disease, contributing to a significant delay between symptoms onset and diagnosis. Even in developed countries, as we observed in the cases described, time to diagnosis can take more than six months, and it represents one of the worst prognostic factors. Back pain is the most common symptom (83-100\% of the patients), and constitutional symptoms, including fever, are relatively rare $(33 \%)$. Spine deformities and neurological deficits are the worst complications of tuberculous spondylitis. Despite all the advances in diagnostic techniques, in developed countries, neurological deficits are still present at the time of diagnosis in $45 \%$ of the cases.

The mean age of patients with tuberculous spondylitis is 45-60 years, although two peaks are reported concerning risk factors: one between 20 and 30 years, related to immigration and HIV, and one between 60 and 70 years, related to immunosuppression and comorbidities. . was suggestive spondylitis. Both patients were operated on by an orthopedic doctor. Anatomical Pathology examination showed tuberculosis spodylitis. The patient was treated with surgical intervention and anti-tuberculosis drug. After surgical intervention, the patient improved clinical symptoms.

Commonly, tuberculous spondylitis affects the thoracic and thoracolumbar segments with initial destruction of the vertebral bodies. Other segments and multifocal involvement are uncommon. Concomitant paraspinal abscesses and epidural involvement can be seen, respectively, in around $70 \%$ and $65 \%$ of the cases. A significant percentage of patients do not show evidence of the primary infection focus. Concurrent pulmonary involvement can range from 2 to $65 \%$. However, in case 2, a paravertebral abscess was found in the cervical area. As in the case described, the patient showed no evidence of a primary infection focus.

Diagnostic suspicion of tuberculous spondylitis is based on clinical and radiological features. Spinal radiography may show a destructive process of vertebras and adjacent discs if osteomyelitis is present. These findings only appear in a late course of the disease and are less pronounced compared to pyogenic infection. Although clinical and radiological findings can be suggestive of tuberculous spondylitis, biopsy-either open biopsy or CT-guided percutaneous aspiration biopsy (PAB) - should be performed in order to obtain a definitive diagnosis. 
The results of the anatomic pathology in both cases showed Tuberculous spondylitis. In the Covid-19 pandemic situation, each patient is subjected to a rapid test. In both cases, the rapid test was negative and had no clinical symptoms of Covid-19. However, surgical procedures that are performed still use level 3 personal protective equipment. Pharmacological treatment should be initiated as soon as the diagnosis is confirmed, with 2 months of HRZE (intensive phase) followed by 10 months of HR (continuation phase).

\section{Conclusions:}

In a covid-19 pandemic situation, diagnosis and management of tuberculous spondylitis must be carried out because it is related to the prognosis of the disease.

\section{References:}

[1] WHO, Global Tuberculosis Report, World Health Organization, Geneva, Switzerland, 2015.

[2] F. Pellisé, "Tuberculosis and Pott's disease, still very relevant health problems," European Spine Journal, vol. 22, no. 4, pp. 527-528, 2013.
[3] Thwaites G et all, British Infection Society guidelinesfor diagnosis and treatment of tuberculosis of CNS in adult and childres, 2009. Sep;59(3):167-87. doi: 10.1016/j.jinf.2009.06.011

[4] A. K. Jain, "Tuberculosis of the spine: a fresh look at an old disease," Journal of Bone and Joint Surgery, vol. 92, no. 7, pp. 905913, 2010.

[5] J. D. Colmenero, J. D Ruiz-Mesa, R. Sanjuan-Jimenez, B. Sobrino, and P. Morata, Establishing the diagnosis of tuberculous vertebral osteomyelitis, European Spine Journal, vol. 22, no. 4, pp. 579-586, 2013.

[6] K. Y. Lee, "Comparison of pyogenic spondylitis and tuberculous spondylitis," Asian Spine Journal, vol. 8, no. 2, pp. 216223, 2014.

[7] A. Rivas-Garcia, S. Sarria-Estrada, C. Torrents-Odin, L. Casas-Gomila, and E. Franquet, "Imaging findings of Pott's disease," European Spine Journal, vol. 22, no. 4, pp. 567-578, 2013. 